\title{
On a Boundary Value Problem for a Polynomial Pencil of the Sturm-Liouville Equation with Spectral Parameter in Boundary Conditions
}

\author{
A. Adiloglu Nabiev \\ Department of Mathematics, Cumhuriyet University, Sivas, Turkey \\ Email: aadiloglu@cumhuriyet.edu.tr
}

How to cite this paper: Adiloglu Nabiev, A. (2016) On a Boundary Value Problem for a Polynomial Pencil of the Sturm-Liouville Equation with Spectral Parameter in Boundary Conditions. Applied Mathematics, 7, 2418-2423.

http://dx.doi.org/10.4236/am.2016.718190

Received: October 25, 2016

Accepted: December 25, 2016

Published: December 28, 2016

Copyright $\odot 2016$ by author and Scientific Research Publishing Inc. This work is licensed under the Creative Commons Attribution International License (CC BY 4.0). http://creativecommons.org/licenses/by/4.0/

\begin{abstract}
The boundary value problem with a spectral parameter in the boundary conditions for a polynomial pencil of the Sturm-Liouville operator is investigated. Using the properties of the transformation operators for such operators, the asymptotic formulas for eigenvalues of the boundary value problem are obtained.
\end{abstract}

\section{Keywords}

Sturm-Liouville Equation, Boundary Value Problem, Transformation Operator, Spectral Theory of Differential Operators, Asymptotic Formulas, Fractional Derivative, Eigenvalue, Eigenfunction, Polynomial Pencil

\section{Introduction}

In this paper the boundary value problem, generated on the finite interval $0 \leq x \leq \pi$ by equation

$$
-y^{\prime \prime}+\left(q_{0}(x)+\lambda q_{1}(x)+\cdots+\lambda^{n-1} q_{n-1}(x)\right) y=\lambda^{2 n} y
$$

and the boundary conditions

$$
P_{n 1}(\lambda) y(0)-y^{\prime}(0)=P_{n 2}(\lambda) y(\pi)+y^{\prime}(\pi)=0
$$

is considered. Here we assume that $n>1, \quad q_{0}(x) \in C[0, \pi]$, $q_{n}(x) \in C^{1}[0, \pi] \quad(k=\overline{1, n-1})$ are complex valued functions; $\lambda$ is a complex parameter and

$$
P_{n j}(\lambda)=i \lambda^{n}+\sum_{k=0}^{n-1} \beta_{k j} \lambda^{k}, j=1 ; 2
$$

with the given constants $\beta_{k j}$. 
It is known that the Sturm-Liouville problems play an important role in solving many problems in mathematical physics. There has been a growing interest in SturmLiouville problems with spectral parameter in boundary conditions in recent years and there are a lot of articles on this subject in the literature. For more detailed analysis we refer to the papers [1]-[9] and the references therein. In the case $n>1$ the simple boundary value problem for the Equation (1) with conditions

$y(0)=y(\pi)=0$ is investigated in [10] (also see [11]).

Note that many of these investigations are based on some integral representations for the fundamental solutions of the Sturm-Liouville equation called transformation operators. The transformation operators for Sturm-Liouville equation and quadratic pencil of the Sturm-Liouville equation are constructed and studied in [12] [13] and [14] [15] respectively, while the corresponding operators for the pencil (1) are investigated in [10] [16].

In this paper using the properties of transformation operators, the considering boundary value problem is investigated and asymptotic formula for the eigenvalues is obtained.

We studied in [10], the solutions $y_{j}(x, \lambda)(j=1,2)$ of the Equation (1) satisfying the initial conditions

$$
y_{j}(0, \lambda)=1, y_{j}^{\prime}(0, \lambda)=(-1)^{j+1} i \lambda^{n}
$$

and it is proved that in the sectors of complex plane

$$
S_{m}=\left\{\lambda: \frac{m \pi}{n} \leq \arg \lambda \leq \frac{(m+1) \pi}{n}\right\}, m=\overline{0,2 n-1}
$$

the solutions $y_{j}(x, \lambda)$ have the following integral representations:

$$
y_{j}(x, \lambda)=\mathrm{e}^{(-1)^{j+1} i \lambda^{n} x}\left[1+\int_{\frac{(-1)^{j+m}-1}{2} x}^{+\infty} K_{v, m}(x, t) \mathrm{e}^{(-1)^{m} 2 i \lambda^{n} t} \mathrm{~d} t\right]
$$

where $v=j+\frac{1}{2}\left[(-1)^{j+m}-(-1)^{j}\right], K_{1, m}(x,),. D_{x} K_{1, m}(x,$.$) and K_{2, m}(x,$.$) ,$

$D_{x} K_{2, m}(x,$.$) belong to L_{1}(-x ;+\infty)$ and $L_{1}(0 ;+\infty)$ respectively. Moreover, if $D_{a, t}^{\alpha} \varphi(x, t)$ denotes Riemann-Liouville fractional derivative of order $\alpha(0<\alpha<1)$ (see [17]) with respect to $t$, i.e.

$$
D_{a, t}^{\alpha} \varphi(x, t) \stackrel{\operatorname{det}}{=} \frac{1}{\Gamma(\alpha)} \frac{\partial}{\partial t} \int_{0}^{t}(t-s)^{\alpha-1} \varphi(x, s) \mathrm{d} s
$$

then for all $x \in[0, \pi]$ the functions $\left(D_{-x, t}^{\frac{1}{n}}\right)^{p} K_{1, n}(x, t)$ and $\left(D_{0, t}^{\frac{1}{n}}\right)^{p} K_{2, m}(x, t)(p=\overline{1, n})$ belong to $L_{1}(-x,+\infty)$ and $L_{2}(0,+\infty)$ respectively. Furthermore, the following equalities are valid: 


$$
\begin{aligned}
\int_{-x}^{+\infty} K_{1, m}(x, t) \mathrm{e}^{(-1)^{m} 2 i \lambda^{n} t} \mathrm{~d} t= & -\sum_{k=0}^{n-1} \gamma_{k+1} \lambda^{-k-1} \alpha_{k}^{(1)}(x) \\
& +(-1)^{m}\left(2 i \lambda^{n}\right)^{-1} \int_{-x}^{+\infty} \mathrm{e}^{(-1)^{m} 2 i \lambda^{n} t}\left(D_{-x, t}^{\frac{1}{n}}\right)^{n} K_{1, m}(x, t) \mathrm{d} t, \\
\int_{0}^{+\infty} K_{2, m}(x, t) \mathrm{e}^{(-1)^{m} 2 i \lambda^{n} t} \mathrm{~d} t & =\sum_{k=0}^{n-1} \gamma_{k+1} \lambda^{-k-1} \alpha_{k}^{(2)}(x) \\
& +(-1)^{m}\left(2 i \lambda^{n}\right)^{-1} \int_{0}^{+\infty} \mathrm{e}^{(-1)^{m} 2 i \lambda^{n} t}\left(D_{0, t}^{\frac{1}{n}}\right)^{n} K_{2, m}(x, t) \mathrm{d} t,
\end{aligned}
$$

where

$$
\begin{gathered}
\gamma_{k}=2^{-\frac{k}{n}} \mathrm{e}^{\frac{i \pi k}{2 n}}, k=\overline{1, n-1}, \\
\alpha_{0}^{(1)}(x)=\alpha_{0}^{(2)}(x)=\gamma_{n-1} \int_{0}^{x} q_{n-1}(s) \mathrm{d} s, \\
\alpha_{k}^{(j)}(x)=\gamma_{n-k-1} \int_{0}^{x} q_{n-k-1}(s) \mathrm{d} s+(-1)^{j} \sum_{p=1}^{k} \gamma_{n-p} \int_{0}^{x} q_{n-p}(s) \alpha_{k-p}^{(j)}(s) \mathrm{d} s, j=1,2 ; k=\overline{1, n-1} .
\end{gathered}
$$

\section{Asymptotic Formulas for the Solutions and Eigenvalues}

By $s(x, \lambda)$ and $c(x, \lambda)$ we denote the solutions of the Equation (1) with initial conditions

$$
s(0, \lambda)=c^{\prime}(0, \lambda)=0, s^{\prime}(0, \lambda)=c(0, \lambda)=1 .
$$

Using integral representations (3) and formulae (4), (5), it is easy to show that for each $\lambda \in S_{m}$

$$
\begin{aligned}
s(x, \lambda)= & \frac{\sin \lambda^{n} x}{\lambda^{n}}+(-1)^{m}\left(2 i \lambda^{n}\right)^{-1} \mathrm{e}^{(-1)^{m} i \lambda^{n} x} \int_{-x}^{\infty} K_{1, m}(x, t) \mathrm{e}^{(-1)^{m} 2 i \lambda^{n} t} \mathrm{~d} t \\
& +(-1)^{m+1}\left(2 i \lambda^{n}\right)^{-1} \mathrm{e}^{(-1)^{m+1} i \lambda^{n} x} \int_{0}^{\infty} K_{2, m}(x, t) \mathrm{e}^{(-1)^{m} 2 i \lambda^{n} t} \mathrm{~d} t \\
s^{\prime}(x, \lambda)= & \cos \lambda^{n} x-\frac{1}{2 i} \sum_{k=0}^{n-1} \gamma_{k+1} \lambda^{-k-1} \alpha_{k}^{(1)}(x) \mathrm{e}^{i \lambda^{n} x}+\frac{1}{2 i} \sum_{k=0}^{n-1} \gamma_{k+1} \lambda^{-k-1} \alpha_{k}^{(2)}(x) \mathrm{e}^{-i \lambda^{n} x} \\
& +(-1)^{m}\left(2 i \lambda^{n}\right)^{-1} \mathrm{e}^{(-1)^{m} i \lambda^{n} x} \int_{-x}^{\infty}\left[D_{x} K_{1, m}(x, t)\right. \\
& \left.-\frac{1}{2}\left(D_{-x, t}^{\frac{1}{n}}\right)^{n} K_{1, m}(x, t)\right] \mathrm{e}^{(-1)^{m} 2 i \lambda^{n} t} \mathrm{~d} t \\
& +(-1)^{m+1}\left(2 i \lambda^{n}\right)^{-1} \mathrm{e}^{(-1)^{m+1} i \lambda^{n} x} \int_{0}^{\infty}\left[D_{x} K_{2, m}(x, t)\right. \\
& \left.+\frac{1}{2}\left(D_{0, t}^{\frac{1}{n}}\right)^{n} K_{2, m}(x, t)\right] \mathrm{e}^{(-1)^{m} 2 i \lambda^{n} t} \mathrm{~d} t,
\end{aligned}
$$




$$
\begin{gathered}
c(x, \lambda)=\cos \lambda^{n} x-\frac{1}{2} \mathrm{e}^{i \lambda^{n} x} \sum_{k=0}^{n-1} \frac{\gamma_{k+1}}{\lambda^{k+1}} \alpha_{k}^{(1)}(x)+\frac{1}{2} \mathrm{e}^{-i \lambda^{n} x} \sum_{k=0}^{n-1} \frac{\gamma_{k+1}}{\lambda^{k+1}} \alpha_{k}^{(2)}(x) \\
+(-1)^{m+1}\left(4 i \lambda^{n}\right)^{-1} \mathrm{e}^{(-1)^{m} i \lambda^{n} x} \int_{-x}^{\infty} \mathrm{e}^{(-1)^{m} 2 i \lambda^{n} t}\left(D_{-x, t}^{\frac{1}{n}}\right)^{n} K_{1, m}(x, t) \mathrm{d} t \\
+(-)^{m+1}\left(4 i \lambda^{n}\right)^{-1} \mathrm{e}^{(-1)^{m+1} i \lambda^{n} x} \int_{0}^{\infty} \mathrm{e}^{(-1)^{m} 2 i \lambda^{n} t}\left(D_{0, t}^{\frac{1}{n}}\right)^{n} K_{2, m}(x, t) \mathrm{d} t, \\
c^{\prime}(x, \lambda)=-\lambda^{n} \sin \lambda^{n} x+\frac{1}{2 i} \mathrm{e}^{i \lambda^{n} x} \sum_{k=0}^{n-1} \gamma_{k+1} \lambda^{n-k-1} \alpha_{k}^{(1)}(x)+\frac{1}{2 i} \mathrm{e}^{-i \lambda^{n} x} \sum_{k=0}^{n-1} \gamma_{k+1} \lambda^{n-k-1} \alpha_{k}^{(2)}(x) \\
+\frac{1}{2} \mathrm{e}^{(-1)^{m+1} i \lambda^{n} x} \int_{0}^{\infty}\left[D_{x} K_{2, m}(x, t)+\frac{1}{2}\left(D_{0, t}^{\frac{1}{n}}\right)^{n} K_{2, m}(x, t)\right] \mathrm{e}^{(-1)^{m} 2 i \lambda^{n} t} \mathrm{~d} t \\
+\frac{1}{2} \mathrm{e}^{(-1)^{m} i \lambda^{n} x} \int_{-x}^{\infty}\left[D_{x} K_{1, m}(x, t)-\frac{1}{2}\left(D_{-x, t}^{\frac{1}{n}}\right)^{n} K_{1, m}(x, t)\right] \mathrm{e}^{(-1)^{m} 2 i \lambda^{n} t} \mathrm{~d} t .
\end{gathered}
$$

Let us consider the boundary problem (1), (2). Denote by $\Delta(\lambda)$ the characteristic function of this problem. Then

$$
\Delta(\lambda)=\left|\begin{array}{ll}
1 & -P_{n 1}(\lambda) \\
P_{n 2}(\lambda) s(\pi, \lambda)+s^{\prime}(\pi, \lambda) & P_{n 2}(\lambda) c(\pi, \lambda)+c^{\prime}(\pi, \lambda)
\end{array}\right|
$$

Zeros of the function $\Delta(\lambda)$ we'll call eigenvalues of the problem (1), (2). Let $w(\lambda, x ; h)$ be the solution of the Equation (1) with initial conditions

$$
w(\lambda, 0 ; h)=1, w^{\prime}(\lambda, 0 ; h)=P_{n 1}(\lambda)
$$

It is clear that

$$
\begin{aligned}
& w(\lambda, x ; h)=P_{n 1}(\lambda) s(x, \lambda)+c(x, \lambda) \\
& w^{\prime}(\lambda, x ; h)=P_{n 1}(\lambda) s^{\prime}(x, \lambda)+c^{\prime}(x, \lambda)
\end{aligned}
$$

and

$$
\Delta(\lambda)=P_{n 2}(\lambda) w(\lambda, \pi ; h)+w^{\prime}(\lambda, \pi ; h)
$$

From formulae (8)-(11) we find that

$$
\begin{aligned}
w(\lambda, \pi ; h)= & P_{n 1}(\lambda) \frac{\sin \lambda^{n} \pi}{\lambda^{n}}+\cos \lambda^{n} \pi-\frac{P_{n 1}(\lambda)+i \lambda^{n}}{2 i \lambda^{n}} \mathrm{e}^{i \lambda^{n} \pi} \sum_{k=0}^{n-1} \lambda^{-k-1} \gamma_{k+1} \alpha_{k}^{(1)}(\pi) \\
& -\frac{P_{n 1}(\lambda)-i \lambda^{n}}{2 i \lambda^{n}} \mathrm{e}^{-i \lambda^{n} \pi} \sum_{k=0}^{n-1} \lambda^{-k-1} \gamma_{k+1} \alpha_{k}^{(2)}(\pi)+o\left(\lambda^{-n}\right) \mathrm{e}^{\left|\operatorname{Im} \lambda^{n} \pi\right|},|\lambda| \rightarrow+\infty \\
w^{\prime}(\lambda, \pi ; h)= & P_{n 1}(\lambda) \cos \lambda^{n} \pi-\lambda^{n} \sin \lambda^{n} \pi-\frac{P_{n 1}(\lambda)-\lambda^{n}}{2 i \lambda^{n}} \mathrm{e}^{i \lambda^{n} \pi} \sum_{k=0}^{n-1} \gamma_{k+1} \lambda^{n-k-1} \alpha_{k}^{(1)}(\pi) \\
& +\frac{P_{n 1}(\lambda)+\lambda^{n}}{2 i \lambda^{n}} \mathrm{e}^{-i \lambda^{n} \pi} \sum_{k=0}^{n-1} \gamma_{k+1} \lambda^{n-k-1} \alpha_{k}^{(2)}(\pi)+o(1) \mathrm{e}^{\left|\operatorname{Im} \lambda^{n} \pi\right|},|\lambda| \rightarrow+\infty
\end{aligned}
$$

Then for $\Delta(\lambda)$ we can write the asymptotic formula

$$
\begin{aligned}
\Delta(\lambda)= & -\lambda^{n} \sin \lambda^{n} \pi+\mathrm{e}^{i \lambda^{n} \pi}\left(i \lambda^{n}+\sum_{k=0}^{n-1} \theta_{k} \lambda^{n-k-1}\right) \\
& +\mathrm{e}^{-i \lambda^{n} \pi}\left(i \lambda^{n}+\sum_{k=0}^{n-1} h_{k} \lambda^{n-k-1}\right)+\mathrm{e}^{\left|\operatorname{Im} \lambda^{n} \pi\right|} o(1),|\lambda| \rightarrow+\infty
\end{aligned}
$$


where $\theta_{k}$ and $h_{k}$ are constants. From this we conclude that there exists the constant $L>0$ such that

$$
\left|\Delta(\lambda)+\lambda^{n} \Delta_{0}(\lambda)\right| \leq L e^{\left|\mathcal{J}_{m} \lambda^{n} \pi\right|}
$$

for all $\lambda$, where

$$
\Delta_{0}(\lambda)=\sin \lambda^{n} \pi-\mathrm{e}^{i \lambda^{n} \pi}\left(i+\sum_{k=0}^{n-2} \lambda^{-(k+1)} \theta_{k}\right)-\mathrm{e}^{-i \lambda^{n} \pi}\left(i+\sum_{k=0}^{n-2} \lambda^{-(k+1)} h_{k}\right)
$$

From (20) we have that for sufficiently large positive integer $k$ there are a finite number of zeros of $\Delta_{0}(\lambda)$ in the circle $O_{k}\left(|\lambda|=\sqrt[n]{k+\frac{1}{2}}\right)$. In other words, the total number of zeros of $\Delta_{0}(\lambda)$ in $O_{k}$ is equal to the total number of zeros of the function $\sin \lambda^{n} \pi$. Moreover, there exists a positive number $N$ such that on the circle $O_{k}\left(|\lambda|=\sqrt[n]{k+\frac{1}{2}}\right)$ the estimation

$$
\left|\lambda^{n} \Delta_{0}(\lambda)\right|>N|\lambda|^{n} \mathrm{e}^{\left|\mathcal{J}_{m} \lambda^{n} \pi\right|}
$$

satisfies. Hence, from (28), (30) and the equality

$$
\Delta(\lambda)=-\lambda^{n} \Delta_{0}(\lambda)+\left(\Delta(\lambda)+\lambda^{n} \Delta_{0}(\lambda)\right)
$$

according to Rouche's theorem we conclude that $\Delta(\lambda)$ and $\lambda^{n} \Delta_{0}(\lambda)$ have the same number of zeros in the circle $O_{k}$ for sufficiently large $k$. Using a simple asymptotic estimations (see [2]), we obtain that zeros having sufficiently large module of the function $\Delta(\lambda)$ lie near rays $\arg \lambda=\frac{m \pi}{n}$, and so the eigenvalues of the problem (1), (2) consist of $2 n$ series. Solving the equation $\Delta(\lambda)=0$ asymptotically we find the following asymptotic formula for $m^{\underline{t h}}$ series of eigenvalues of the problem (1), (2):

$$
\lambda_{k, m}=\mathrm{e}^{\frac{2 i m \pi}{n}} \sqrt[n]{k}+\sum_{s=1}^{n} \frac{b_{s}^{(m)}}{k^{1+\frac{s-1}{n}}}+0\left(\frac{1}{k^{1+\frac{n-1}{n}}}\right), k \rightarrow+\infty
$$

where $\sup _{s}\left|b_{s}^{(m)}\right|<\infty$.

Theorem 2. Boundary value problem (1), (2) has a countable number of eigenvalues. The eigenvalues having sufficiently large module are placed near the rays $\arg \lambda=\frac{m \pi}{n}(m=\overline{0, m-1})$, and $m^{\underline{t h}}$ series of these satisfy the asymptotic formula (23).

\section{References}

[1] Fulton, C.T. (1977) Two-Point Boundary Value Problems with Eigenvalue Parameter in the Boundary Conditions. Proceedings of the Royal Society of Edinburgh, 77A, 293-308. https://doi.org/10.1017/S030821050002521X

[2] Mukhtarov, O.Sh. (1994) Discontinuous Boundary-Value Problem with Spectral Parameter in Boundary Conditions. Turkish Journal of Mathematics, 18, 183-192. 
[3] Altinisik, N., Kadakal, M. and Mukhtarov, O.Sh. (2004) Eigenvalues and Eigenfunctions of Discontinuous Sturm-Liouville Problems with Eigenparameter-Dependent Boundary Conditions. Acta Mathematica Hungarica, 102, 159-193. https://doi.org/10.1023/B:AMHU.0000023214.99631.52

[4] Wang, A., Sun, J., Hao, X. and Yao, S. (2009) Completeness of Eigenfunctions of SturmLiouville Problems with Transmission Conditions. Methods and Applications of Analysis, 16, 299-312. https://doi.org/10.1016/j.jmaa.2008.08.008

[5] Hinton, D.B. (1979) An Expansion Theorem for an Eigenvalue Problem with Eigenvalue Parameter in the Boundary Conditions. Quarterly Journal of Mathematics. Oxford Journals, 30, 33-42. https://doi.org/10.1093/qmath/30.1.33

[6] Binding, P., Hyrnyv, R. and Langer, H. (2001) Ellipitic Eigenvalue Problem with Eigenparameter Dependent Boundary Conditions. Journal of Differential Equation, 174, 30-54. https://doi.org/10.1006/jdeq.2000.3945

[7] Rasulov, M.L. (1967) Methods of Countour Integration. North Holland Publishing Co.

[8] Shkalikov, A.A. (1982) Boundary Value Problems for Ordinary Differential Equations with a Parameter in Boundary Conditions. Functional Analysis and Its Applications, 16, 324236.

[9] Mamedov, Kh.R. (2010) On an Inverse Scattering Problem for a Discontinuous SturmLiouville Equation with a Spectral Parameter in the Boundary Condition. Boundary Value Problems, 2010, Article ID: 171967. https://doi.org/10.1155/2010/171967

[10] Guseinov, I.M., Nabiev, A.A. and Pashayev, R.T. (2000) Transformation Operators and Asymptotic Formulas for the Eigenvalues of a Polynomial Pencil of Sturm-Liouville Operators. Siberian Journal of Mathematics, 41, 453-464. https://doi.org/10.1007/BF02674102

[11] Agamaliyev, A. and Nabiyev, A. (2005) On Eigenvalues of Some Boundary Value Problems for a Polynomial Pencil of Sturm-Liouville Equation. Applied Mathematics and Computations, 165, 503-505. https://doi.org/10.1016/j.amc.2004.04.116

[12] Marchenko, V.A. (1997) Sturm-Liouville's Operators and Their Application. Kiev.

[13] Povzner, A.Ya. (1948) On Differential Equations of Type Sturm-Liouville on the Semi Axis. Mathematical Surveys (Mat. Sbornik), 23, 3-52.

[14] Jaulent, M. and Jean, C. (1972) On an Inverse Scattering Problem with an Energy-Dependent Potential. Annales de P Institut Henri Poincaré A, 17, 363-378.

[15] Guseinov, G.Sh. (1985) On Spectral Analisys of Quatratic Pencil of Sturm-Liouville Operators. Doklady Akademii Nauk SSSR, 285, 1292-1296.

[16] Guseinov, I.M. (1997) On a Transformation Operator. Journal of Mathematical Notes, 62, 206-215. https://doi.org/10.1007/bf02355905

[17] Samko, S.G., Kilbas, A.A. and Marichev, O.M. (1987) Integral and Derifatives of Fractional Order and Its Applications. Minsk, Nauka and Tekhnika. 
Submit or recommend next manuscript to SCIRP and we will provide best service for you:

Accepting pre-submission inquiries through Email, Facebook, LinkedIn, Twitter, etc. A wide selection of journals (inclusive of 9 subjects, more than 200 journals)

Providing 24-hour high-quality service

User-friendly online submission system

Fair and swift peer-review system

Efficient typesetting and proofreading procedure

Display of the result of downloads and visits, as well as the number of cited articles

Maximum dissemination of your research work

Submit your manuscript at: http://papersubmission.scirp.org/

Or contact am@scirp.org 